PROCEEDINGS OF THE

AMERICAN MATHEMATICAL SOCIETY

Volume 125, Number 8, August 1997, Pages 2327-2335

S 0002-9939(97)03853-7

\title{
EQUIVALENCE OF SOME CONTRACTIVITY PROPERTIES OVER METRICAL STRUCTURES
}

\author{
JACEK R. JACHYMSKI
}

(Communicated by Palle E. T. Jorgensen)

\begin{abstract}
We establish an equivalence between eight contractive definitions. Next, we formulate a separation theorem for right upper semicontinuous functions. As its application, we give a complete characterization of relations between fixed point theorems of Boyd and Wong (1969), and Browder (1968).
\end{abstract}

\section{INTRODUCTION}

In his paper [16] Tasković formulated the so-called monotone fixed point principle with the intention to subsume a number of other fixed point results, in particular, the ones of Browder (see [2] or [4], p. 18), Dugundji and Granas [3], and Krasnoselskij et al. (see [10] or [4], p. 13). However, recently, Turinici [17] showed that a basic lemma used in [16] was false, and indicated some conditions, which suffice for the validity of the lemma and the principle. Then, he observed that the above results cannot be derived, in the way proposed by Tasković, from such a modified principle. In this connection he posed the question of whether or not this is possible via different procedures.

The main result of this paper establishes an equivalence between eight contractivity properties (cf. Theorem 1). Among these, two are of special interest, namely (e) (the strong Boyd-Wong contractivity) and (g) (the strong Matkowski contractivity). If we consider their general counterparts

(E) there exists a right upper semicontinuous function $\phi: \mathbf{R}_{+} \mapsto \mathbf{R}_{+}$such that $T$ is $\phi$-contractive,

and, respectively

$(\mathrm{G})$ there exists an increasing function $\phi: \mathbf{R}_{+} \mapsto \mathbf{R}_{+}$such that (2) holds and $T$ is $\phi$-contractive,

then it follows from our Theorem 1 that we have the relations

$$
(\mathrm{E}) \Rightarrow(\mathrm{e}) \Leftrightarrow(i) \quad \text { and } \quad(\mathrm{G}) \Rightarrow(\mathrm{g}) \Leftrightarrow(i), \quad \text { where } i \in\{a, \ldots, h\} .
$$

In particular, the mutually equivalent fixed point principles related to (a)-(h) are methodologically reducible to the one due to Matkowski [11], but not vice versa (cf. Theorem 5). This solves the open question posed by Turinici [17]. As a further

Received by the editors July 3, 1995 and, in revised form, September 19, 1995 and February 14, 1996.

1991 Mathematics Subject Classification. Primary 47H10, 54H25.

Key words and phrases. Upper semicontinuous function, increasing function, cluster point, nonlinear contraction, iteration.

(C) 1997 American Mathematical Society 
consequence, Theorem 3.3 in [4, p. 13] by Krasnoselskij does follow from Theorem 3.2 in $[4$, p. 12] due to Matkowski. This corrects a remark in [4, p. 13].

In Section 4 we show that Theorem 1 of Boyd and Wong [1] essentially improves the results of Browder [2]; moreover, our Theorems 3 and 4 give a complete characterization of the relations between these theorems. In particular, the implication $(\mathrm{e}) \Rightarrow(\mathrm{E})$ is false in general. As a by-product of our Theorem 2, we obtain that Theorem 1 of [1] is equivalent to its simplified version given by Mukherjea ([14], Theorem 1.3). On the other hand conditions (E) and $(\mathrm{G})$ are independent, which means that the Boyd-Wong principle is not in general reducible to Matkowski's, and the reciprocal is also false in general (cf. Remark 3).

Finally, in Section 6 we prove among others that if

$$
M_{+}(\phi):=\left\{t>0: \lim _{s \rightarrow t^{+}} \phi(s)=t\right\},
$$

where $\phi$ is an increasing function from $\mathbf{R}_{+}$, the nonnegative reals, into $\mathbf{R}_{+}$, such that

$$
\lim _{n \rightarrow \infty} \phi^{n}(t)=0, \quad \text { for all } t \in \mathbf{R}_{+},
$$

then the set $M_{+}(\phi)$ is at most countable. This settles, in the negative, another question posed by Turinici in [17].

\section{Equivalent CONTRactivity PRoperties}

Given a function $\phi: \mathbf{R}_{+} \mapsto \mathbf{R}_{+}$such that $\phi(t)<t$ for $t>0$, and a selfmap $T$ of a metric space $(X, d)$, we say that $T$ is $\phi$-contractive if

$$
d(T x, T y) \leq \phi(d(x, y)), \quad \text { for all } x, y \in X .
$$

The following theorem establishes the equivalence between some contractivity properties. Each of conditions (a)-(h) of Theorem 1 is sufficient for the existence of a fixed point if the metric space considered is complete.

Theorem 1. Let $T$ be a selfmap of a metric space $(X, d)$. The following statements are equivalent.

(a) (Browder [2]) There exists an increasing and right continuous function $\phi: \mathbf{R}_{+} \mapsto \mathbf{R}_{+}$such that $T$ is $\phi$-contractive.

(b) (Dugundji-Granas [3]) There exists a map $\Theta: X \times X \mapsto \mathbf{R}_{+}$with $\inf \{\Theta(x, y)$ : $a \leq d(x, y) \leq b\}>0$, for $a, b>0$, such that

$$
d(T x, T y) \leq d(x, y)-\Theta(x, y), \quad \text { for all } x, y \in X .
$$

(c) (Krasnoselskij et al. [10]) There exists a map $\Gamma: X \times X \mapsto \mathbf{R}_{+}$with $\sup \{\Gamma(x, y)$ : $a \leq d(x, y) \leq b\}<1$, for $a, b>0$, such that

$$
d(T x, T y) \leq \Gamma(x, y) d(x, y), \quad \text { for all } x, y \in X .
$$

(d) (Krasnoselskij-Stetsenko [9]) There exists a continuous function $\psi: \mathbf{R}_{+} \mapsto$ $\mathbf{R}_{+}$with $\psi(t)>0$ for $t>0$, such that

$$
d(T x, T y) \leq d(x, y)-\psi(d(x, y)), \quad \text { for all } x, y \in X .
$$

(e) (cf. Boyd-Wong [1]) There exists an upper semicontinuous function $\phi: \mathbf{R}_{+} \mapsto$ $\mathbf{R}_{+}$such that $T$ is $\phi$-contractive.

(f) There exists a function $\phi: \mathbf{R}_{+} \mapsto \mathbf{R}_{+}$with $\lim \sup _{s \rightarrow t} \phi(s)<t$, for all $t>0$, such that $T$ is $\phi$-contractive. 
(g) (cf. Matkowski [11]) There exists a strictly increasing function $\phi: \mathbf{R}_{+} \mapsto \mathbf{R}_{+}$ such that (2) holds and $T$ is $\phi$-contractive.

(h) There exists a strictly increasing and continuous function $\phi: \mathbf{R}_{+} \mapsto \mathbf{R}_{+}$such that $T$ is $\phi$-contractive.

Remark 1. In Theorem 1 (e) is a specialization of the condition due to Boyd and Wong [1] in which, originally, $\phi$ is right upper semicontinuous. Similarly, (g) is a variant of Matkowski's condition [11], which involves a function $\phi$ not necessarily strictly monotonic.

The proof of Theorem 1 depends on

Lemma 1. Assume that $0 \leq a<b \leq \infty$ and let the function $\phi: \mathbf{R}_{+} \mapsto \mathbf{R}_{+}$be such that $\phi(t)<t$ for $t>0$, as well as

$$
\begin{array}{ll}
\limsup _{s \rightarrow t} \phi(s)<t & \text { for } t \in(a, b), \text { and } \\
\limsup _{s \rightarrow a^{+}} \phi(s)<a & \text { if } a>0 .
\end{array}
$$

Then, there exists a strictly increasing and continuous function $\psi:[a, b) \mapsto \mathbf{R}_{+}$ such that

$$
\phi(t) \leq \psi(t)<t \quad \text { for } t \in[a, b) \cap(0, \infty) .
$$

Proof. We shall consider two cases.

$\left.1^{\circ}\right) a>0$. Let $\left\{b_{n}\right\}$ be a strictly increasing sequence, with $b_{0}:=a$ and $b_{n} \rightarrow b$. For $n \in \mathbf{N}$ define

$$
\alpha_{n}:=\sup \left\{\frac{\phi(t)}{t}: t \in\left[a, b_{n}\right]\right\} .
$$

By the admitted hypotheses, $\alpha_{n}<1$, for $n \in \mathbf{N}$. Put $\alpha:=\sup _{n \in N} \alpha_{n}$. Without loss we may assume $\alpha=1$ and (passing to a subsequence if necessary) $\left\{\alpha_{n}\right\}$ is strictly increasing. Define the function $\psi:[a, b) \mapsto \mathbf{R}_{+}$as

$$
\operatorname{graph}(\psi):=\text { the polygonal line with nodes }\left\{\left(b_{n}, \alpha_{n+1} b_{n}\right): n \in \mathbf{N} \cup\{0\}\right\} .
$$

It is easy to verify that $\psi$ has all the required properties.

$\left.2^{\circ}\right) a=0$. Let $\left\{a_{n}\right\}$ and $\left\{b_{n}\right\}$ be two sequences converging to 0 and $b$ respectively, with

$$
0<a_{n+1}<a_{n}<b_{n}<b_{n+1}<b, \quad \text { for } n \in \mathbf{N} .
$$

Put $\alpha_{n}:=\sup \left\{\phi(t) / t: t \in\left[a_{n}, b_{n}\right]\right\}$ for all $n$. As in case $\left.1^{\circ}\right)$, we have $\alpha_{n}<1$, $n \in \mathbf{N}$. Denote also $\alpha:=\sup _{n \in N} \alpha_{n}$. Without loss, assume $\alpha=1$ and $\left\{\alpha_{n}\right\}$ is strictly increasing. Define the sequences $\left\{r_{k}\right\}_{k=1}^{\infty}$ and $\left\{n_{k}\right\}_{k=0}^{\infty}$ by putting $n_{0}:=0$ and for $k \in \mathbf{N}$,

$$
\begin{aligned}
& r_{k}:=\max \left\{\alpha_{n+1} a_{n}: n>n_{k-1}\right\}, \\
& n_{k}:=\max \left\{n>n_{k-1}: \alpha_{n+1} a_{n}=r_{k}\right\} .
\end{aligned}
$$

We now introduce a function $\psi: \mathbf{R}_{+} \mapsto \mathbf{R}_{+}$as follows. Put $\psi(0):=0$ and $\psi(t):=$ $\alpha_{n_{1}+1} t$, for $t \in\left[a_{n_{1}}, b_{n_{1}}\right]$. Further, define $\psi$ on $\left[b_{n_{1}}, \infty\right)$ as in case $1^{\circ}$ ) (with $a:=b_{n_{1}}$ ). And, on $\left(0, a_{n_{1}}\right]$ define $\psi$ as

$$
\operatorname{graph}(\psi):=\text { the polygonal line with nodes }\left\{\left(a_{n_{k}}, r_{k}\right): k \in \mathbf{N}\right\} .
$$

We leave it to the reader to verify that $\psi$ has all the properties we need. 
Proof of Theorem 1. We shall verify the implications (b) $\Leftrightarrow(\mathrm{c}),(\mathrm{d}) \Leftrightarrow(\mathrm{e}),(\mathrm{h}) \Rightarrow$ (a) $\Rightarrow(\mathrm{e}) \Rightarrow(\mathrm{f}) \Rightarrow(\mathrm{h}),(\mathrm{h}) \Rightarrow(\mathrm{g}) \Rightarrow(\mathrm{f})$ and $(\mathrm{h}) \Rightarrow(\mathrm{c}) \Rightarrow(\mathrm{f})$.

The relation (b) $\Leftrightarrow$ (c) was proved in [3]. (d) $\Rightarrow$ (e) follows with $\phi(t)=t-\psi(t)$, $t \in \mathbf{R}_{+}$, and (e) $\Rightarrow(\mathrm{d})$ with a result in Michael [13]. Further, (h) $\Rightarrow(\mathrm{a})$ is trivial, as well as (e) $\Rightarrow(\mathrm{f})$; moreover, (a) $\Rightarrow$ (e) is clear (each increasing function is left upper semicontinuous) and (f) $\Rightarrow(\mathrm{h})$ results from Lemma 1. Further, $(\mathrm{h}) \Rightarrow(\mathrm{g})$ holds in the way described by [2], and $(\mathrm{g}) \Rightarrow(\mathrm{f})$ follows from Theorem 5 . Finally, assume (h) is true. Define a map $\Gamma: X \times X \mapsto \mathbf{R}_{+}$by

$$
\Gamma(x, x):=0, \quad x \in X, \quad \text { and } \quad \Gamma(x, y):=\frac{d(T x, T y)}{d(x, y)} \quad \text { for } x \neq y .
$$

Let $a, b>0, a<b$. Since $T$ is $\phi$-contractive,

$$
\sup \{\Gamma(x, y): a \leq d(x, y) \leq b\} \leq \sup \left\{\frac{\phi(t)}{t}: a \leq t \leq b\right\}<1
$$

(because the function $t \mapsto \phi(t) / t$ attains its maximum on $[a, b]$ ); so, (e) holds. To prove (c) $\Rightarrow$ (f) define, for $n \in \mathbf{N}$

$$
A_{n}:=\left\{(x, y) \in X \times X: \frac{1}{n} \leq d(x, y) \leq n\right\} .
$$

These sets are nonempty for sufficiently large $n$, say $n \geq n_{0}$. Put, for all such $n$

$$
\alpha_{n}:=\sup \left\{\frac{d(T x, T y)}{d(x, y)}:(x, y) \in A_{n}\right\} .
$$

Let $\phi: \mathbf{R}_{+} \mapsto \mathbf{R}_{+}$be defined as $\phi(0):=0, \phi(t):=\alpha_{n_{0}} t$ for $t \in\left[1 / n_{0}, n_{0}\right]$ and $\phi(t):=\alpha_{n} t$ for $t \in[1 / n, 1 /(n-1)) \cup(n-1, n], n>n_{0}$. It is easy to see that (f) is verified.

\section{A SEPARATION THEOREM FOR RIGHT UPPER SEMICONTINUOUS FUNCTIONS}

For each function $\phi: \mathbf{R}_{+} \mapsto \mathbf{R}_{+}$with $\phi(t)<t$ for $t>0$ denote

$$
M_{-}(\phi):=\left\{t>0: \limsup _{s \rightarrow t_{-}} \phi(s)=t\right\},
$$

as well as (for $t>0)$

$$
S_{\phi}(t):=\inf M_{-}(\phi) \cap(t, \infty)
$$

(with the convention inf $\emptyset=\infty$ ).

Theorem 2. Let a function $\phi: \mathbf{R}_{+} \mapsto \mathbf{R}_{+}$be right upper semicontinuous and $\phi(t)<t$ for $t>0$. Then, there exists a right continuous function $\psi: \mathbf{R}_{+} \mapsto \mathbf{R}_{+}$ such that

$$
\phi(t) \leq \psi(t)<t \quad \text { for } t>0 .
$$

Moreover,

1. If $M_{-}(\phi)=\emptyset$, then $\psi$ may be assumed to be continuous and strictly increasing on $\mathbf{R}_{+}$.

2. If $M_{-}(\phi) \neq \emptyset$, then for $t \in M_{-}(\phi)$ we have $S_{\phi}(t)>t$ and either $S_{\phi}(t) \in$ $M_{-}(\phi)$, or $S_{\phi}(t)=\infty$. (Hence, $t \mapsto S_{\phi}(t)$ is the immediate successor map of $\left(M_{-}(\phi) \cup\{\infty\}, \leq\right)$ and, as such, $M_{-}(\phi)$ is at most countable.) Furthermore, 
(a) if $a:=\inf M_{-}(\phi)>0$, then $a \in M_{-}(\phi), \mathbf{R}_{+}=[0, a) \cup \bigcup_{t \in M_{-}(\phi)}\left[t, S_{\phi}(t)\right)$ and $\psi$ may be assumed to be continuous and strictly increasing on each interval in this decomposition;

(b) if $\inf M_{-}(\phi)=0$, then $(0, \infty)=\bigcup_{t \in M_{-}(\phi)}\left[t, S_{\phi}(t)\right)$ and $\psi$ may be assumed to be continuous and strictly increasing on each interval in this decomposition.

Proof. Assume that $M_{-}(\phi)=\emptyset$. Hence and by the right upper semicontinuity, we infer that $\limsup _{s \rightarrow t} \phi(s)<t$ for $t>0$. To get the required function $\psi$, it suffices to apply Lemma 1 putting in it $a=0$ and $b=\infty$.

In the sequel we assume that $M_{-}(\phi) \neq \emptyset$. To show that for $t \in M_{-}(\phi), S(t)>t$ and $S(t) \in M_{-}(\phi)$ if $S(t)$ is finite, it suffices to prove that the set $M_{-}(\phi)$ has no rightside cluster points in $(0, \infty)$. Suppose, on the contrary, there is an $r, r>0$, and a sequence $\left\{t_{n}\right\}$ such that $t_{n} \in M_{-}(\phi)$, and $t_{n} \rightarrow r^{+}$. By (3), there is a sequence $\left\{s_{n}\right\}$ such that $r<s_{n}<t_{n}$ and $\left|\phi\left(s_{n}\right)-t_{n}\right| \rightarrow 0$. This implies $\limsup _{s \rightarrow r^{+}} \phi(s)=$ $r$, a contradiction, since by hypothesis $\lim \sup _{s \rightarrow r^{+}} \phi(s) \leq \phi(r)<r$. A similar argument can be used in order to show that, on the other hand, each leftside cluster point of the set $M_{-}(\phi)$ belongs to it. This fact easily implies that

$$
\bigcup_{t \in M_{-}(\phi)}[t, S(t)) \supseteq\left(\inf M_{-}(\phi), \infty\right) .
$$

Clearly, the intervals $[t, S(t))\left(t \in M_{-}(\phi)\right)$ are disjoint. So we may apply Lemma 1 to define a function $\psi$ on the set $\bigcup_{t \in M_{-}(\phi)}[t, S(t))$. Now, if $a:=$ $\inf M_{-}(\phi)>0$, then $a \in M_{-}(\phi)$, so we may conclude from (4) that $\bigcup_{t \in M_{-}(\phi)}[t, S(t))$ $=[a, \infty)$ and it suffices to apply again Lemma 1 to define $\psi$ on $[0, a)$. In the case in which $a=0,(4)$ implies that $\bigcup_{t \in M_{-}(\phi)}[t, S(t))=(0, \infty)$ and it suffices to put $\psi(0):=0$ to get the desirable function.

Remark 2. By Theorem 2, Theorem 1 of Boyd and Wong [1] is equivalent to Theorem 1.3 of Mukherjea [14].

\section{Comparison of the Boyd-Wong and Browder theorems}

Boyd and Wong [1] have given an example to show that their Theorem 1 does improve an earlier result of Rakotch [15]. However, one can easily redefine the function $\psi$ of this example to fulfill condition (a) of Theorem 1, which means that, in this case, Browder's theorem [2] can be applied. The following result explains completely the relations between these theorems. In particular, Theorem 1 in [1] appears to be essentially more general than Theorem 1 in [2].

Theorem 3. Let a function $\phi: \mathbf{R}_{+} \mapsto \mathbf{R}_{+}$be right upper semicontinuous and such that $\phi(t)<t$ for $t>0$. Define the set $M_{-}(\phi)$ as in (3). The following statements are equivalent.

(i) $M_{-}(\phi)=\emptyset$.

(ii) Given a metric space $(X, d)$ and a $\phi$-contractive map $T: X \mapsto X$, there exists an increasing and right continuous function $\psi: \mathbf{R}_{+} \mapsto \mathbf{R}_{+}$such that $T$ is $\psi$-contractive.

Proof. (i) $\Rightarrow$ (ii). Assume that a map $T: X \mapsto X$ is $\phi$-contractive. By Theorem 2, there exists a continuous and increasing function $\psi: \mathbf{R}_{+} \mapsto \mathbf{R}_{+}$such that $\phi(t) \leq$ $\psi(t)<t$ for $t>0$, which immediately implies that $T$ is $\psi$-contractive. 
(ii) $\Rightarrow$ (i). Suppose, on the contrary, that $M_{-}(\phi) \neq \emptyset$. Then, there exists a strictly increasing sequence $\left\{t_{n}\right\}$ and a positive real $t_{0}$ such that $t_{n} \rightarrow t_{0}$ and $\phi\left(t_{n}\right) \rightarrow t_{0}$. By passing to a subsequence if necessary, we may assume that $\phi\left(t_{n+1}\right)>t_{n}$ for $n \in \mathbf{N}$. Define

$$
X:=\left\{t_{n}: n \in \mathbf{N}\right\}, \quad d\left(t_{n}, t_{m}\right):=\max \left\{t_{n}, t_{m}\right\} \text { if } n \neq m, \text { and } d\left(t_{n}, t_{n}\right):=0 .
$$

Then $(X, d)$ is a metric space ( $d$ is said to be an ultrametric or non-Archimedean metric; see, e.g., [5], p. 504). Further, let

$$
T\left(t_{1}\right):=t_{1} \quad \text { and } \quad T\left(t_{n+1}\right):=t_{n} \quad \text { for } n \in \mathbf{N} .
$$

If $m, n \in \mathbf{N}$ and $m>n$, then $d\left(T\left(t_{n}\right), T\left(t_{m}\right)\right)=t_{m-1}<\phi\left(t_{m}\right)=\phi\left(d\left(t_{n}, t_{m}\right)\right)$ so $T$ is $\phi$-contractive. By hypothesis, $T$ is $\psi$-contractive with the function $\psi$ being increasing and right continuous. Then, for $x \in X$,

$$
d\left(T^{n} x, t_{1}\right)=d\left(T^{n} x, T^{n} t_{1}\right) \leq \psi^{n}\left(d\left(x, t_{1}\right)\right) \leq \psi^{n}\left(t_{0}\right),
$$

which implies that $T^{n} x \rightarrow t_{1}$ uniformly for $x \in X$. But this is impossible, since $d\left(T^{n} t_{n+2}, t_{1}\right)=t_{2}>0$. Therefore, the set $M_{-}(\phi)$ is empty.

By Theorem 3, for any right upper semicontinuous function $\phi: \mathbf{R}_{+} \mapsto \mathbf{R}_{+}$such that the set $M_{-}(\phi)$ is nonempty, there is a $\phi$-contractive map, for which Browder's theorem cannot be applied. Our next result deals with such functions. Precisely, some functions $\phi$ may be so "bad" that for certain $\phi$-contractive maps $T$, there is no equivalent metric $\rho$ such that $T$ be $\psi$-contractive in $(X, \rho)$ with $\psi$ increasing and right continuous.

Theorem 4. Let a function $\phi: \mathbf{R}_{+} \mapsto \mathbf{R}_{+}$be right upper semicontinuous and such that $\phi(t)<t$ for $t>0$. Assume that the set $M_{-}(\phi)$ defined by (3) is nonempty. The following statements are equivalent.

(i) $\inf M_{-}(\phi)>0$.

(ii) Given a complete metric space $(X, d)$ and a $\phi$-contractive map $T: X \mapsto X$, there exist an equivalent metric $\rho$, and an increasing and right continuous function $\eta: \mathbf{R}_{+} \mapsto \mathbf{R}_{+}$such that $T$ is $\eta$-contractive in $(X, \rho)$.

Proof. (i) $\Rightarrow$ (ii). Assume that the map $T: X \mapsto X$ is $\phi$-contractive. By Theorem 2, there exists a right continuous function $\psi$ such that $\phi(t) \leq \psi(t)<t$ for $t>0$, and $\psi$ is strictly increasing and continuous on $[0, a)$, where $a:=\inf M_{-}(\phi)$. By Theorem 1 of [1], $T$ has a fixed point $x_{0}$ and $T^{n} x \rightarrow x_{0}$ for all $x \in X$. Furthermore, if $d\left(x, x_{0}\right)<r<a$, then

$$
d\left(T^{n} x, x_{0}\right) \leq \psi^{n}\left(d\left(x, x_{0}\right)\right) \leq \psi^{n}(r) .
$$

Hence, we may conclude that $T^{n} x \rightarrow x_{0}$ uniformly for $x \in B\left(x_{0}, r\right)$, the open ball about $x_{0}$ with the radius $r$. By Meyers' theorem [12], there is an equivalent metric $\rho$ such that $\rho(T x, T y) \leq \frac{1}{2} \rho(x, y)$. So it suffices to put $\eta(t):=\frac{1}{2} t$ for $t \in \mathbf{R}_{+}$.

(ii) $\Rightarrow$ (i). Suppose, on the contrary, that $\inf M_{-}(\phi)=0$. Then, there exist a strictly decreasing sequence $\left\{t_{k}\right\}_{k=1}^{\infty}$ and strictly increasing sequences $\left\{t_{n}^{(k)}\right\}_{n=1}^{\infty}$ $(k \in \mathbf{N})$ such that

$$
\begin{aligned}
& t_{k} \in M_{-}(\phi), \quad t_{k} \rightarrow 0, \quad t_{n}^{(k)} \rightarrow t_{k} \text { as } n \rightarrow \infty(k \in \mathbf{N}), \\
& \phi\left(t_{n}^{(k)}\right) \rightarrow t_{k} \quad \text { as } n \rightarrow \infty(k \in \mathbf{N}), \\
& \phi\left(t_{n+1}^{(k)}\right)>t_{n}^{(k)} \quad \text { and } \quad \phi\left(t_{n}^{(k)}\right)>t_{k+1} \quad \text { for } k, n \in \mathbf{N}
\end{aligned}
$$


(cf. the proof of Theorem 3, (ii) $\Rightarrow(\mathrm{i})$ ). Define the set $X$ by

$$
X:=\left\{t_{n}^{(k)}: k, n \in \mathbf{N}\right\} \cup\{0\} .
$$

Let $d(x, y):=\max \{x, y\}$ for $x, y \in X, x \neq y$, and $d(x, x):=0$. Then $(X, d)$ is a complete metric space. Define the map $T$ by

$$
T(0):=0, \quad T\left(t_{n+1}^{(k)}\right):=t_{n}^{(k)} \quad \text { and } \quad T\left(t_{1}^{(k)}\right):=t_{1}^{(k+1)} \quad \text { for } k, n \in \mathbf{N} .
$$

Then $T$ is $\phi$-contractive as the reader can routinely verify. By hypothesis, there is an equivalent metric $\rho$, and a right continuous and increasing function $\eta$ : $\mathbf{R}_{+} \mapsto \mathbf{R}_{+}$ such that

$$
\rho(T x, T y) \leq \eta(\rho(x, y)), \quad \text { for all } x, y \in X .
$$

Then, there is a positive real $r$ such that $d(x, 0)<r$ implies $\rho(x, 0)<1$, for $x \in X$. Since $t_{k} \rightarrow 0$ and $t_{n}^{(k)}<t_{k}$, there is a $k_{0}$ in $\mathbf{N}$ such that $t_{n}^{\left(k_{0}\right)}<r$ for $n \in \mathbf{N}$. Then, $d\left(t_{n}^{\left(k_{0}\right)}, 0\right)<r$ so $\rho\left(t_{n}^{\left(k_{0}\right)}, 0\right)<1$. By (5) and (6), we get

$$
0<\rho\left(t_{1}^{\left(k_{0}\right)}, 0\right)=\rho\left(T^{n}\left(t_{n+1}^{\left(k_{0}\right)}\right), T^{n}(0)\right) \leq \eta^{n}\left(\rho\left(t_{n+1}^{\left(k_{0}\right)}, 0\right)\right) \leq \eta^{n}(1), \quad \text { for } n \in \mathbf{N},
$$

which yields a contradiction, since $\eta^{n}(1) \rightarrow 0$.

\section{Comparison of the Browder And Matkowski theorems}

The following result is a counterpart of Theorem 3 for the case in which one compares the theorems of Browder [2] and Matkowski [11].

Theorem 5. Let a function $\phi: \mathbf{R}_{+} \mapsto \mathbf{R}_{+}$be increasing and such that (2) holds. Let the set $M_{+}(\phi)$ be defined by (1). The following statements are equivalent.

(i) $M_{+}(\phi)=\emptyset$.

(ii) Given a metric space $(X, d)$ and a $\phi$-contractive map $T$, there exists an increasing and right continuous function $\psi: \mathbf{R}_{+} \mapsto \mathbf{R}_{+}$such that $T$ is $\psi$-contractive.

Proof. (i) $\Rightarrow$ (ii). By the hypothesis, $\lim _{s \rightarrow t^{+}} \phi(s)<t$ for $t>0$, and by monotonicity, $\lim _{s \rightarrow t^{-}} \phi(s) \leq \phi(t)<t$ for $t>0$. Consequently, $\lim _{s \rightarrow t} \phi(s)<t$ for $t>0$. That (ii) holds now follows from the implication (f) $\Rightarrow$ (a) of Theorem 1.

(ii) $\Rightarrow$ (i). Suppose, on the contrary, there is a $t_{0}$ in $(0, \infty)$ such that $\lim _{t \rightarrow t_{0}^{+}} \phi(t)$ $=t_{0}$. Define $X:=\mathbf{R}_{+}$and for $x, y \in X, d(x, y):=\max \{x, y\}$ if $x \neq y$, and $d(x, x):=0$. Further, let $T:=\phi$. By monotonicity, we have that $d(T x, T y)=$ $\phi(d(x, y))$. So by (ii), $T$ is $\psi$-contractive with $\psi$ increasing and right continuous. In particular, for any $x \in \mathbf{R}_{+}$,

$$
\psi(x)=\psi(d(x, 0)) \geq d(T x, T 0)=\phi(x)
$$

which yields

$$
\psi\left(t_{0}\right)=\lim _{t \rightarrow t_{0}^{+}} \psi(t) \geq \lim _{t \rightarrow t_{0}^{+}} \phi(t)=t_{0},
$$

a contradiction, since $\psi\left(t_{0}\right)<t_{0}$.

The counterpart of Theorem 4 is quite different. Namely, we have

Theorem 6. Let a function $\phi$ be as in Theorem 5. Then, given a complete metric space $(X, d)$ and a $\phi$-contractive map $T: X \mapsto X$, there exists an equivalent metric $\rho$ such that $T$ is a Banach contraction in $(X, \rho)$. 
Proof. By Theorem 1.2 of [11], $T$ has a fixed point $x_{0}$ and $T^{n} x \rightarrow x_{0}$ for all $x \in X$. Furthermore, for any $r>0$,

$$
d\left(T^{n} x, x_{0}\right)=d\left(T^{n} x, T^{n} x_{0}\right) \leq \psi^{n}(r) \quad \text { if } d\left(x, x_{0}\right)<r,
$$

which forces the uniform convergence $T^{n} x \rightarrow x_{0}$ with respect to $x$ satisfying $d\left(x, x_{0}\right)<r$. So the result follows from Meyers' theorem [12].

Remark 3. Example 1 in [6] shows that Matkowski's theorem is not reducible to the Boyd-Wong principle. The reciprocal is also false as can be deduced from the proof of Theorem 4, (ii) $\Rightarrow$ (i). A unified approach to both theorems, involving the Meir-Keeler type conditions, has been presented in [8]. Finally, let us note that Matkowski's theorem is an immediate consequence of an iff fixed point criterion given in [7].

\section{A characterization of the Set $M_{+}(\phi)$}

In this section we answer negatively the question posed by Turinici in [17].

Theorem 7. Let a function $\phi: \mathbf{R}_{+} \mapsto \mathbf{R}_{+}$be increasing and such that (2) is satisfied. Let the set $M_{+}(\phi)$ be defined by (1). Then the following statements hold.

$1^{\circ}$. For any $t \in M_{+}(\phi)$, there is a $\delta, \delta>0$, such that the function $\left.\phi\right|_{(t, t+\delta)}$ is constant.

$2^{\circ} .0$ is the only possible cluster point of $M_{+}(\phi)$.

$3^{\circ}$. The set $M_{+}(\phi)$ is at most countable.

$4^{\circ}$. If $\phi$ is strictly increasing, then $M_{+}(\phi)=\emptyset$.

Proof. Conditions $3^{\circ}$ and $4^{\circ}$ are immediate consequences of $2^{\circ}$ and $1^{\circ}$, respectively $\left(3^{\circ}\right.$ also follows from $\left.1^{\circ}\right)$. To prove $1^{\circ}$, fix a $t_{0}$ in $M_{+}(\phi)$. By monotonicity, $\phi(t) \geq$ $\lim _{s \rightarrow t_{0}^{+}} \phi(s)=t_{0}$, for $t>t_{0}$. We shall show that for some $\delta>0, \phi(t)=t_{0}$ for $t \in\left(t_{0}, t_{0}+\delta\right)$. Suppose, on the contrary, there is a sequence $\left\{t_{n}\right\}$ such that $t_{n} \searrow t_{0}$ and $\phi\left(t_{n}\right)>t_{0}$. By monotonicity, we may conclude that $\phi(t)>t_{0}$ for $t>t_{0}$, and hence $\phi^{n}(t)>t_{0}$ for all such $t$ and all $n \in \mathbf{N}$, which contradicts (2).

Observe that $1^{\circ}$ easily implies that $M_{+}(\phi)$ has no rightside cluster points in $(0, \infty)$. So to prove $2^{\circ}$, it suffices to show that $M_{+}(\phi)$ has no leftside cluster points. Suppose not. Then, there exist a $t_{0}>0$ and a sequence $\left\{t_{n}\right\}$ such that $t_{n} \in M_{+}(\phi)$ and $t_{n} \nearrow t_{0}$, which forces $\lim _{s \rightarrow t_{0}^{-}} \phi(s)=t_{0}$, a contradiction, since by monotonicity, $\lim _{s \rightarrow t_{0}^{-}} \phi(s) \leq \phi\left(t_{0}\right)<t_{0}$.

\section{ACKNOWLEDGEMENT}

I am grateful to the referee for a number of useful suggestions.

\section{REFERENCES}

1. D. W. Boyd and J. S. W. Wong, On nonlinear contractions, Proc. Amer. Math. Soc. 20 (1969), 458-464. MR 39:916

2. F. Browder, On the convergence of successive approximations for nonlinear functional equations, Indag. Math. 30 (1968), 27-35. MR 37:5743

3. J. Dugundji and A. Granas, Weakly contractive maps and elementary domain invariance theorems, Bull. Greek Math. Soc. 19 (1978), 141-151. MR 80d:54035

4. J. Dugundji and A. Granas, Fixed point theory. I, Polish Scientific Publishers, Warszawa 1982. MR 83j:54038

5. R. Engelking, General topology, Polish Scientific Publishers, Warszawa 1977. MR 58:18316b 
6. J. Jachymski, A generalization of the theorem by Rhoades and Watson for contractive type mappings, Math. Japon. 38 (1993), 1095-1102. MR 94k:54076

7. _ An iff fixed point criterion for continuous self-mappings on a complete metric space, Aequationes Math. 48 (1994), 163-170. MR 96c:54069

8. __ Equivalent conditions and the Meir-Keeler type theorems, J. Math. Anal. Appl. 194 (1995), 293-303. MR 96h:54033

9. M. A. Krasnoselskij and V. J. Stetsenko, About the theory of equations with concave operators, Sib. Mat. Zh. 10 (1969), 565-572 (in Russian).

10. M. A. Krasnoselskij, G. M. Vainikko et al., Approximate solution of operator equations, Wolters Noordhoff, Groningen 1972. MR 52:6515

11. J. Matkowski, Integrable solutions of functional equations, Diss. Math. 127 (1975). MR 54:772

12. P. R. Meyers, A converse to Banach's contraction theorem, J. Res. Nat. Bur. Standards Sect. 71 B (1967), 73-76. MR 36:4521

13. E. Michael, Continuous selections. I, Ann. of Math. 63 (1956), 361-382. MR 17:990e

14. A. Mukherjea, Contractions and completely continuous mappings, Nonlinear Anal. T.M.A. 1 (1977), 235-247. MR 58:31030

15. E. Rakotch, A note on contractive mappings, Proc. Amer. Math. Soc. 13 (1962), 459-465. MR 26:5555

16. M. R. Tasković, A monotone principle of fixed points, Proc. Amer. Math. Soc. 94 (1985), 427-432. MR 87c:54065

17. M. Turinici, The monotone principle of fixed points: a correction, Proc. Amer. Math. Soc. 122 (1994), 643-645. CMP 94:15

Institute of Mathematics, Technical University of Łódź, Żwirki 36, 90-924 Łódź, POLAND

E-mail address: jachymsk@lodz1.p.lodz.pl 